\title{
Two-dimensional NMR lineshape analysis of single, multiple, zero and double quantum correlation experiments
}

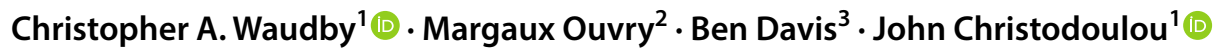

Received: 29 September 2019 / Accepted: 31 December 2019 / Published online: 8 January 2020

(c) The Author(s) 2020

\begin{abstract}
NMR spectroscopy provides a powerful approach for the characterisation of chemical exchange and molecular interactions by analysis of series of experiments acquired over the course of a titration measurement. The appearance of NMR resonances undergoing chemical exchange depends on the frequency difference relative to the rate of exchange, and in the case of one-dimensional experiments chemical exchange regimes are well established and well known. However, two-dimensional experiments present additional complexity, as at least one additional frequency difference must be considered. Here we provide a systematic classification of chemical exchange regimes in two-dimensional NMR spectra. We highlight important differences between exchange in HSQC and HMQC experiments, that on a practical level result in more severe exchange broadening in HMQC spectra, but show that complementary alternatives to the HMQC are available in the form of HZQC and HDQC experiments. We present the longitudinal relaxation optimised SOFAST-H(Z/D)QC experiment for the simultaneous acquisition of sensitivity-enhanced HZQC and HDQC spectra, and the longitudinal and transverse relaxation optimised BEST-ZQ-TROSY for analysis of large molecular weight systems. We describe the application of these experiments to the characterisation of the interaction between the Hsp90 N-terminal domain and a small molecule ligand, and show that the independent analysis of HSQC, HMQC, HZQC and HDQC experiments provides improved confidence in the fitted dissociation constant and dissociation rate. Joint analysis of such data may provide improved sensitivity to detect and analyse more complex multi-state interaction mechanisms such as induced fit or conformational selection.
\end{abstract}

Keywords Chemical exchange $\cdot$ Double-quantum $\cdot$ Lineshape analysis $\cdot$ Multiple-quantum $\cdot$ Titrations $\cdot$ Two-dimensional NMR $\cdot$ Zero-quantum

\section{Introduction}

The NMR lineshape (i.e. intensity, frequency, phase and linewidth) of a spin undergoing chemical exchange (i.e. a spin that is interconverting between multiple states in dynamic equilibrium) is well known to provide a powerful

Electronic supplementary material The online version of this article (https://doi.org/10.1007/s10858-019-00297-7) contains supplementary material, which is available to authorized users.

Christopher A. Waudby

c.waudby@ucl.ac.uk

1 Institute of Structural and Molecular Biology, University College London and Birkbeck College, London, UK

2 Biotechnologie, Sanofi, Route d'Avignon, 30390 Aramon, France

3 Vernalis (R\&D) Limited, Granta Park, Great Abington, UK spectroscopic probe of the underlying exchange process. This lineshape may be modulated by a variety of means, including rf pulse sequences such as CPMG pulse trains (Gopalan et al. 2018), $R_{1 \rho}$ spin locks (Massi and Peng 2018; Xue 2015) or saturation with a single frequency or frequency comb (Vallurupalli et al. 2012; Fawzi et al. 2011; Yuwen et al. 2018); field shuttling (Cousin 2016); or variation of external parameters such as protein or ligand concentration or temperature (Waudby et al. 2016, 2019). The resulting modulations may then be fitted to determine details of the exchange process such as chemical shift differences, populations of states, and the rate of exchange between them. As such, NMR spectroscopy provide an indispensable tool for studying both intramolecular dynamics and biomolecular and other host-guest interactions, without the need for extrinsic labels such as fluorophores (Palmer 2014; Schneider et al. 2018; Pons and Millet 2001; Kovermann et al. 2016). 
The effects of chemical exchange on lineshapes in one-dimensional NMR spectra are well understood (Bain 2003). Depending on the frequency difference, $\Delta \omega$, between two resonances, $\mathrm{A}$ and $\mathrm{B}$, that are in exchange with rate $k_{\mathrm{ex}}=k_{\mathrm{AB}}+k_{\mathrm{BA}}$ (where $k_{\mathrm{AB}}$ and $k_{\mathrm{BA}}$ are the forward and backward rates respectively), slow and fast exchange regimes may be defined that have characteristic limiting behaviour. We do not distinguish in this work between the slow and slow-intermediate or fast and fast-intermediate regimes, which instead are encompassed within our definitions of the slow and fast exchange limits. Differences in tranverse relaxation rates, $\Delta R_{2}$, may also affect the observed exchange regime, and therefore $k_{\mathrm{ex}}$ should strictly be compared to $\left|\Delta \omega+i \Delta R_{2}\right|$ instead of $|\Delta \omega|$. The effect of this additional term is to shift exchange towards a slower regime than otherwise expected, but in the majority of situations relevant to biomolecular titrations the frequency difference is likely to remain the dominant term. Therefore, for simplicity hereonin we will assume that there is no significant difference in relaxation rates between states (although any such differences can still be treated exactly by numerical analyses).

In the slow exchange regime $\left(k_{\mathrm{ex}} \ll|\Delta \omega|\right)$, resonance intensities are modulated according to their population (plus a lifetime line broadening contribution to the linewidth) but no chemical shift perturbations are observed (to first order in $\left.k_{\text {ex }}\right)$. In contrast, in the fast exchange regime $\left(k_{\text {ex }} \ll|\Delta \omega|\right)$ a single resonance is observed at a population weighted average of the original chemical shifts, and with an exchangeinduced contribution to the linewidth determined by both the frequency difference between states and the exchange rate (discussed further below). Lastly, when the exchange rate and frequency difference are comparable, severe line broadening can result (the 'intermediate exchange' regime). For equal populations of the exchanging states $\mathrm{A}$ and B, this is best characterised by the 'coalescence point' at which the two original resonances can no longer be distinguished (defined by the vanishing first and second derivatives of the lineshape), which occurs when $|\Delta \omega|=\sqrt{2} k_{\text {ex }}$ (Bain 2003). Alternatively, exchange regimes may be characterised by the dependence of the exchange-induced line broadening term $R_{\text {ex }}$ on the static magnetic field strength, $B_{0}$, through the parameter $\alpha=d\left(\ln R_{\mathrm{ex}}\right) / d\left(\ln B_{0}\right)$ which varies from 0 to 2 between the slow and fast exchange limits (Palmer et al. 2001).

As the appearance of one-dimensional NMR spectra can be modulated strongly by the chemical exchange process, observed spectra may be fitted quantitatively to numerical solutions of the Bloch-McConnell equations governing the evolution of magnetisation vectors (or at a more sophisticated level of theory, to the Liouville-von Neumann equation for the time evolution of the quantum mechanical density operator) in order to determine the microscopic rate constants for the exchange process, and the populations and chemical shifts of the various states (McConnell 1958; Binsch 1969). This procedure, termed 'lineshape analysis' or 'dynamic NMR', is particularly effective when a series of one-dimensional spectra can be analysed as a function of an external parameter such as temperature or ligand concentration, in order to determine parameters such as activation energies and dissociation constants.

The analysis of interactions within more complex macromolecules, such as proteins and nucleic acids, requires the use of two-dimensional NMR experiments such as the HSQC (heteronuclear single quantum correlation) (Bodenhausen and Ruben 1980) or HMQC (heteronuclear multiple quantum correlation) (Bax et al. 1983) to resolve the hundreds of resonances that arise from component residues. While in some cases it is possible to perform one-dimensional lineshape analysis on cross-sections from such spectra (Greenwood 2011; Günther and Schaffhausen 2002), this approach risks a number of systematic errors (Waudby et al. 2016). Indeed, exchange of transverse magnetisation during preparation, chemical shift evolution and mixing periods has recently been shown to give rise to coherent cross-peaks that cannot be accounted for by one-dimensional treatments (Waudby et al. 2019). Instead, we have previously developed a truly two-dimensional lineshape analysis strategy, based on fitting observed two-dimensional spectra to complete quantum mechanical simulations of the underlying pulse sequence (Waudby et al. 2016). The analysis has been implemented in a software tool, TITAN, which has since been applied to study a wide range of biomolecular interactions (Danhart 2017; McShan 2018; Srb 2019).

Two-dimensional lineshape analysis has two particular differences from the well-known one-dimensional theory. Firstly, there are now two chemical shift differences, $\Delta \delta_{I}$ and $\Delta \delta_{S}$, to consider and therefore it is not always possible to classify resonances as being simply in fast or slow exchange regimes. Secondly, in experiments such as the HMQC, chemical shift evolution occurs as a combination of zero and double quantum coherences, with frequencies $\omega_{S} \pm \omega_{I}$, and it is the differences in these frequencies between states that should be compared to the exchange rate rather than the difference in single quantum frequencies. Indeed, we remarked in our original work on two-dimensional lineshape analysis that HSQC and HMQC experiments can give rise to different patterns of exchange broadening that must therefore be analysed with the appropriate framework (Waudby et al. 2016).

In this work, we expand on these earlier observations, by presenting a systematic classification of chemical exchange regimes appropriate for two-dimensional NMR experiments. We also consider zero- and double-quantum correlation experiments (HZQC and HDQC respectively), and describe new sensitivity-optimised pulse sequences for their acquisition that may offer a number of advantages over more commonly used HSQC and HMQC experiments. 


\section{Results}

\section{Classification of exchange regimes in two-dimensional NMR experiments}

In this work, we consider heteronuclear correlation spectra involving two spins, labelled $I$ and $S$, undergoing chemical exchange between two states, $\mathrm{A}$ and $\mathrm{B}$, with rate $k_{\mathrm{ex}}=k_{\mathrm{AB}}+k_{\mathrm{BA}}$. We will also assume that the difference in relaxation rates between states is negligible relative to the frequency difference, i.e. $\Delta R_{2} \ll|\Delta \omega|$. It is then convenient to define the relative frequency difference $\xi_{X}=\Delta \omega_{X} / k_{\text {ex }}$ (where $X$ denotes spin $I$ or $S$ ), such that $|\xi| \ll 1$ represents the fast exchange limit and $|\xi| \gg 1$ represents the slow exchange limit (in a one-dimensional sense). The canonical coalesence point $|\Delta \omega|=\sqrt{2} k_{\text {ex }}$ therefore corresponds to $|\xi|=\sqrt{2}$ (Bain 2003).

We will focus in particular on the analysis of molecular interactions using NMR titration measurements, exemplified by the interaction of a protein, $\mathrm{P}$, with a ligand, $\mathrm{L}$, to form a complex, PL:

$\mathrm{P}+\mathrm{L} \underset{k_{\mathrm{off}}}{\stackrel{k_{\mathrm{on}}}{\rightleftharpoons}} \mathrm{PL}$

where the dissociation constant $K_{\mathrm{d}}=k_{\text {off }} / k_{\text {on }}$. As the free ligand concentration, [L], always increases as ligand is added, the exchange rate, $k_{\mathrm{ex}}=k_{\mathrm{on}}[\mathrm{L}]+k_{\text {off }}$, must also increase monotically with the fraction bound, $p_{\mathrm{B}}$, such that $k_{\text {ex }}=k_{\text {off }} /\left(1-p_{\mathrm{B}}\right)$ (Peng et al. 2001). The relative frequency difference, $\xi=\Delta \omega / k_{\mathrm{ex}}=\left(1-p_{\mathrm{B}}\right) \Delta \omega / k_{\mathrm{off}}$, therefore decreases as the titration proceeds, and so it is not always possible to classify a spin system as being in a single exchange regime throughout a titration. However, as the effects of chemical exchange on resonance lineshapes are most strongly manifest at the titration midpoint $\left(\mathrm{p}_{B}=1 / 2\right)$, we will generally use the relative frequency differences at this point, $\xi=\Delta \omega / 2 k_{\text {off }}$, as a convenient point of reference in discussions below.

\section{Single quantum correlation experiments}

Single quantum (SQ) correlation experiments, such as the HSQC or (amide) TROSY experiments (Bodenhausen and Ruben 1980; Pervushin et al. 1997), have two characteristic parameters, $\xi_{I}$ and $\xi_{S}$, corresponding to the frequency differences in direct and indirect dimensions. Four twodimensional exchange regimes may therefore be identified: fast exchange in both dimensions (FF, $\left|\xi_{I}\right| \ll 1$ and $\left|\xi_{S}\right| \ll 1$ ); slow exchange in both dimensions (SS, $\left|\xi_{I}\right| \gg 1$ and $\left|\xi_{S}\right| \gg 1$ ); fast exchange in the direct dimension and slow exchange in the indirect dimension $\left(\mathrm{FS},\left|\xi_{I}\right| \ll 1\right.$ and
$\left|\xi_{S}\right| \gg 1$ ); and lastly, slow exchange in the direct dimension and fast exchange in the indirect dimension (SF, $\left|\xi_{I}\right| \gg 1$ and $\left.\left|\xi_{S}\right| \ll 1\right)$. These regimes are marked schematically on a set of frequency axes in Fig. 1a. 'Fast exchange', in the sense of $2 \mathrm{D}$ resonances progressively changing frequency along the course of a titration, will only be observed within the 'FF' region where exchange is fast with respect to all relevant frequency differences.

When ligand is added, the observed perturbation $\Delta \omega_{\mathrm{obs}}$ to the frequency of the single resonance observed in the fast exchange limit, or to the frequency of the resonance of state A observed in the slow exchange limit, and the exchange contribution to the resonance linewidth, $R_{\mathrm{ex}}$, have been calculated for the indirect dimension to second order in $\xi$ and $\xi^{-1}$ for the fast and slow exchange limits respectively (see SI text). The resulting expressions recapitulate generally well known results (Peng et al. 2001) and are presented in Table 1 for later comparison. In the fast exchange limit the observed frequency is simply the population weighted average of the individual states, while in the slow exchange limit a much smaller frequency perturbation is observed, that is only second order in the (relatively slow) exchange rate and inversely proportional to the (relatively large) frequency difference between the exchanging states. Conversely, in fast exchange the exchange-induced line broadening is second order in the relatively small frequency difference between the exchanging states, and inversely proportional to the relatively large exchange rate, and therefore will also generally be small in magnitude. The exchange contribution to the linewidth in slow exchange is determined by the lifetime line broadening, and is therefore more significant for the minor state where the populations are highly skewed. Corresponding relations for the direct dimension can be obtained by exchanging $S$ and $I$ terms. As the directly detected dimension is always a single quantum coherence, this is the same for all other experiments discussed below.

To illustrate these exchange regimes, a series of 2D spectra have been simulated according to a titration of a protein with a ligand, interacting through a simple two-state mechanism as discussed above. Ligand concentrations were chosen (relative to an arbitrary $K_{\mathrm{d}}$ ) to give bound populations varying uniformly from 0 to $99.9 \%$. Chemical shift differences were defined according to the relative frequency difference at the titration midpoint, $\xi=\Delta \omega / 2 k_{\text {off }}$, to represent the four $2 \mathrm{D}$ exchange regimes identified above. The simulated spectra are plotted in Fig. 1b-e, and the corresponding relative frequency differences, decreasing along the course of the titration, are marked on Fig. 1a. From these simulations, we observe that exchange in the 'SS' and 'FF' regimes (Fig. 1c, d respectively) appears as might be expected from $1 \mathrm{D}$ analogues. However, in the mixed 'FS' and 'SF' regimes more complex behaviour is observed, in which progressive 


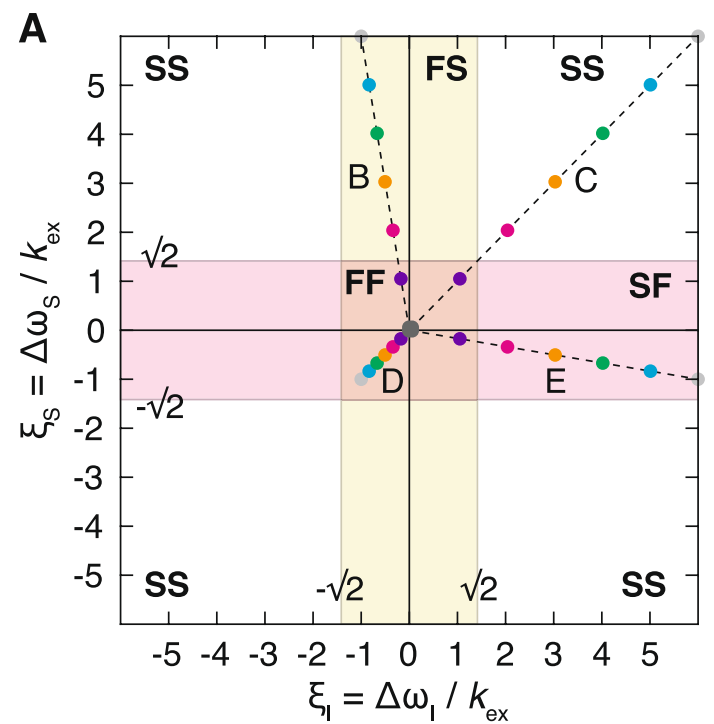

Fig. 1 Classification and examples of two-dimensional exchange regimes for single-quantum correlation experiments. a Two-dimensional exchange regimes are plotted as a function of the relative frequency differences $\xi_{I}=\Delta \omega_{I} / k_{\mathrm{ex}}$ and $\xi_{S}=\Delta \omega_{S} / k_{\mathrm{ex}}$. A yellow band indicates the region of fast exchange in the direct dimension (bounded by the coalescence point), while the red band indicates the region of fast exchange in the indirect dimension. b-e Simulated titration measurements for residues having relative frequency differences (at

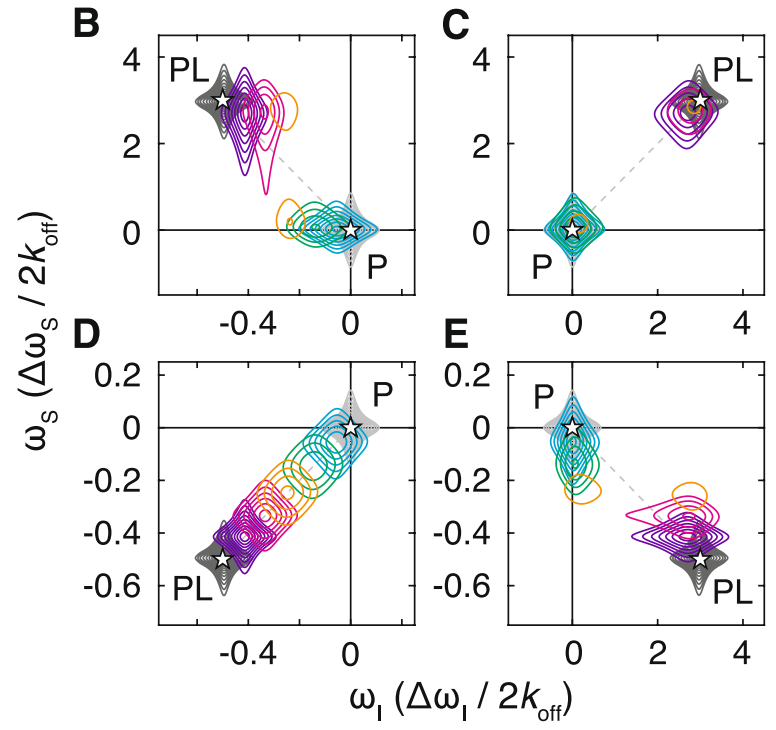

the titration midpoint): $\mathbf{b} \xi_{I}=0.5$ and $\xi_{S}=3 ; \mathbf{c} \xi_{I}=3$ and $\xi_{S}=3$; d $\xi_{I}=0.5$ and $\xi_{S}=0.5$; and $\mathbf{e} \xi_{I}=3$ and $\xi_{S}=0.5$. Asterisks mark the positions of the free and bound resonances. Ligand concentrations were selected so that the bound fraction varied uniformly from 0 to 99.9\% (grey-blue-purple-grey). Contour levels are equal for all spectra, spaced at intervals incrementing $1.4 \times$ from a base value of $2 \%$ of the maximum intensity in the apo spectrum. The relative frequency differences, varying across the titration series, are plotted in a

Table 1 Observed perturbations to the frequency, $\Delta \omega$, and linewidth, $R_{\mathrm{ex}}$, of the single resonance observed in the fast exchange limit, or of the resonance of state $\mathrm{A}$ in the slow exchange limit, calculated for single, multiple, zero and double quantum evolution periods

\begin{tabular}{llllll}
\hline Exchange regime & Term & SQ & MQ & ZQ & DQ \\
\hline Slow & $\Delta \omega_{\text {obs }}$ & $\frac{p_{\mathrm{A}} p_{\mathrm{B}} k_{\mathrm{ex}}^{2}}{\Delta \omega_{\mathrm{S}}}$ & $\frac{p_{\mathrm{A}} p_{\mathrm{B}} k_{\mathrm{ex}}^{2} \Delta \omega_{\mathrm{S}}}{\Delta \omega_{\mathrm{S}}^{2}-\Delta \omega_{\mathrm{I}}^{2}}$ & $\frac{p_{\mathrm{A}} p_{\mathrm{B}} k_{\mathrm{ex}}^{2}}{\Delta \omega_{\mathrm{S}}-\Delta \omega_{\mathrm{I}}}$ & $\frac{p_{\mathrm{A}} p_{\mathrm{B}} k_{\mathrm{ex}}^{2}}{\Delta \omega_{\mathrm{S}}+\Delta \omega_{\mathrm{I}}}$ \\
& $R_{\mathrm{ex}}$ & $k_{\mathrm{AB}}$ & $k_{\mathrm{AB}}$ & $k_{\mathrm{AB}}$ & $k_{\mathrm{AB}}$ \\
Fast & $\Delta \omega_{\mathrm{obs}}$ & $p_{\mathrm{B}} \Delta \omega_{\mathrm{S}}$ & $p_{\mathrm{B}} \Delta \omega_{\mathrm{S}}$ & $p_{\mathrm{B}}\left(\Delta \omega_{\mathrm{S}}-\Delta \omega_{\mathrm{I}}\right)$ & $p_{\mathrm{B}}\left(\Delta \omega_{\mathrm{S}}+\Delta \omega_{\mathrm{I}}\right)$ \\
& $R_{\mathrm{ex}}$ & $\frac{p_{\mathrm{A}} p_{\mathrm{B}} \Delta \omega_{\mathrm{S}}^{2}}{k_{\mathrm{ex}}}$ & $\frac{p_{\mathrm{A}} p_{\mathrm{B}}\left(\Delta \omega_{\mathrm{S}}^{2}+\Delta \omega_{\mathrm{I}}^{2}\right)}{k_{\mathrm{ex}}}$ & $\frac{p_{\mathrm{A}} p_{\mathrm{B}}\left(\Delta \omega_{\mathrm{S}}-\Delta \omega_{\mathrm{I}}\right)^{2}}{k_{\mathrm{ex}}}$ & $\frac{p_{\mathrm{A}} p_{\mathrm{B}}\left(\Delta \omega_{\mathrm{S}}+\Delta \omega_{\mathrm{I}}\right)^{2}}{k_{\mathrm{ex}}}$ \\
\hline
\end{tabular}

chemical shift changes are observed along the 'fast' dimension only (Fig. 1b, e).

\section{Zero and double quantum correlation experiments}

Having examined exchange regimes within the HSQC experiment, and as a prelude to the analysis of the more complicated HMQC experiment, we have considered exchange within zero and double quantum correlation experiments. Although not commonly acquired, amide zero quantum coherences have favourable relaxation rates, intermediate between single or multiple quantum coherences and the TROSY coherence $H^{\beta} N^{ \pm}$(Pervushin et al. 1999), while for methyl spin systems sharper lines can be obtained in HZQC spectra than in the standard 'methyl-TROSY' HMQC experiment itself (Tugarinov et al. 2004). Zero and double quantum correlation experiments have also been suggested as a means of alleviating exchange broadening in residues undergoing conformational exchange (Pervushin 2001).

The analysis of HZQC and HDQC experiments is essentially identical to that of the HSQC, with the difference in single quantum frequencies, $\xi_{S}$, replaced by the difference in zero or double quantum frequencies, $\xi_{S} \pm \xi_{I}$, as appropriate. Therefore, four two-dimensional exchange regimes can be identified (Fig. 2a, b) as described above for the HSQC (Fig. 1a) (essentially, the chemical shift difference ' $y$ ' axis is skewed at $\pm 45^{\circ}$ relative to the conventional single quantum axis). The observed frequency perturbations and exchange contribution to relaxation rates in the indirect dimensions have again been calculated (see SI text) and are tabulated for slow and fast exchange limits in Table 1. 

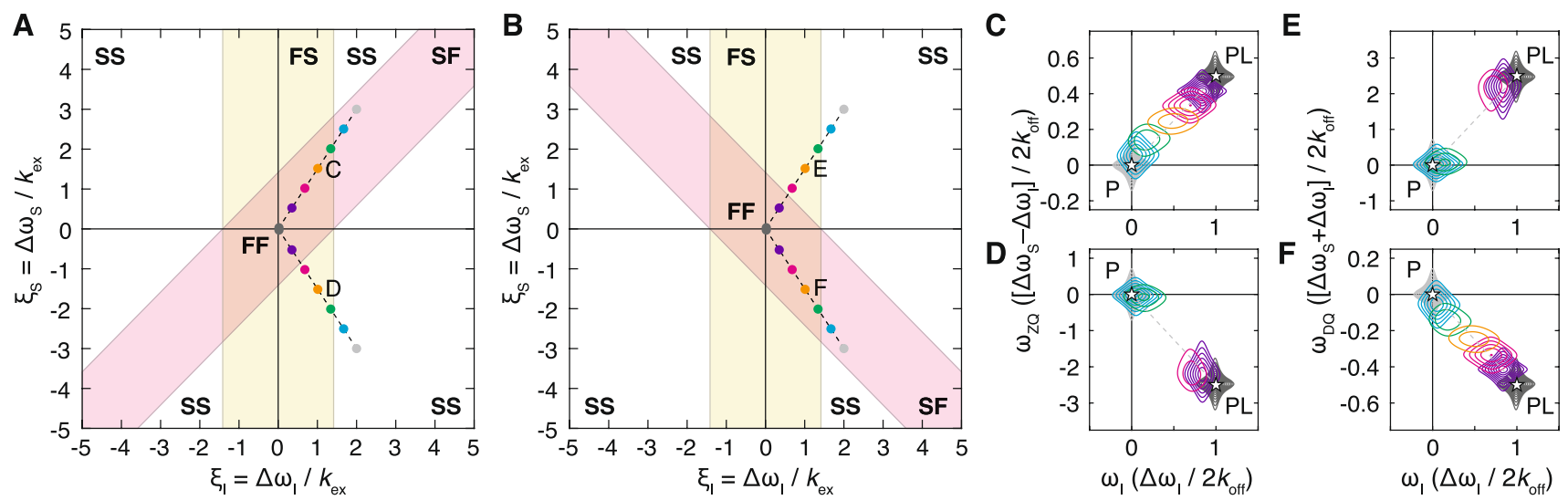

Fig. 2 Classification and examples of two-dimensional exchange regimes for zero and double quantum correlation experiments. a, b Two-dimensional exchange regimes for a zero quantum and $\mathbf{b}$ double quantum correlation experiments are plotted as a function of the relative frequency differences $\xi_{I}=\Delta \omega_{I} / k_{\mathrm{ex}}$ and $\xi_{S}=\Delta \omega_{S} / k_{\mathrm{ex}}$. Yellow bands indicate the region of fast exchange in the direct dimension (bounded by the coalescence point), while red bands indicate the region of fast exchange in the indirect dimension, corresponding to the differences in zero or double quantum frequencies. c-f Simulated

It may be observed that the ranges of parameter space within the fast exchange (FF) regime for HZQC and HDQC experiments are complementary (Fig. 2a, b). This has two practical implications. Firstly, a resonance that is in the FS exchange regime within one experiment may be within the FF regime in the other, and this may facilitate tracking chemical shift changes during the course of a titration. This is illustrated for the simulated titration spectra shown in Fig. 2c-f. Secondly, due to the differences in exchange regimes between these experiments, two-dimensional lineshape analysis conducted on both sets of experiments should prove complementary, and their independent analysis may provide an additional layer of validation on results obtained from such fitting.

\section{Multiple quantum correlation experiments}

In the HMQC experiment, magnetisation evolves as a mixture of zero and double quantum coherences during $t_{1}$ (Bax et al. 1983). Thus, in contrast to the HSQC, there are three characteristic parameters to be considered (relative the to exchange rate): the frequency difference, $\xi_{I}$, in the direct dimension, and the differences in both the zero and double quantum frequencies, $\xi_{S} \pm \xi_{I}$, in the indirect dimension. From this, seven two-dimensional exchange regimes may therefore be identified, corresponding to fast and slow exchange in the direct dimension, and fast and slow exchange with respect to both zero and double quantum frequency differences, as indicated in Fig. 3a (note that an eighth case, slow exchange in the direct dimension and fast exchange
HZQC and HDQC titration measurements for residues having relative frequency differences (at the titration midpoint): $\mathbf{c}, \mathbf{e} \xi_{I}=1$ and $\xi_{S}=1.5 ; \mathbf{d}, \mathbf{f} \xi_{I}=1$ and $\xi_{S}=-1.5$. Asterisks mark the positions of the free and bound resonances. Ligand concentrations were selected so that the bound fraction varied uniformly from 0 to $99.9 \%$ (greyblue-purple-grey). Contour levels are equal for all spectra, spaced at intervals incrementing $1.4 \times$ from a base value of $2 \%$ of the maximum intensity in the apo spectrum. The relative frequency differences, varying across the titration series, are plotted in $\mathbf{a}$ and $\mathbf{b}$

with respect to zero and double quantum frequency differences, is not possible). 'Fast exchange' behaviour of 2D resonances will only be observed within the 'FFF' regime, in which exchange is fast with respect to all frequency differences. This is a smaller region of parameter space than for the single quantum experiment (Fig. 1a), with the practical consequence that during titrations it is likely to be more difficult to follow progressive chemical shift changes using HMQC experiments than using HSQC experiments.

As for the HSQC experiment, the observed chemical shift perturbations and the exchange contribution to the resonance line broadening in the indirect dimension have been calculated for the slow and fast zero/double quantum exchange regimes and are tabulated in Table 1 . The most significant difference between the HSQC and HMQC experiments is that chemical shift changes and line broadening in the indirect dimension now depend upon the frequency difference in the direct dimension also. Two observations are particularly striking. Firstly, the exchange contribution to line broadening, $R_{\mathrm{ex}}$, in the fast $\left(\mathrm{F}_{\mathrm{Z}} \mathrm{F}_{\mathrm{D}}\right)$ exchange regime scales as $\Delta \omega_{S}^{2}+\Delta \omega_{I}^{2}$, rather than $\Delta \omega_{S}^{2}$ as in the single quantum case. Thus, HMQC resonances are subject to more significant line broadening than in the equivalent HSQC experiment. This may have practical consequences for the ability to detect and track chemical shift changes over the course of a titration experiment. Secondly, chemical shift perturbations in the slow $\left(\mathrm{S}_{\mathrm{Z}} \mathrm{S}_{\mathrm{D}}\right)$ exchange regime are proportional to $\Delta \omega_{S} /\left(\Delta \omega_{S}^{2}-\Delta \omega_{I}^{2}\right)$. When the chemical shift difference in the direct dimension is large this term can become close to zero or even change sign, such that the 

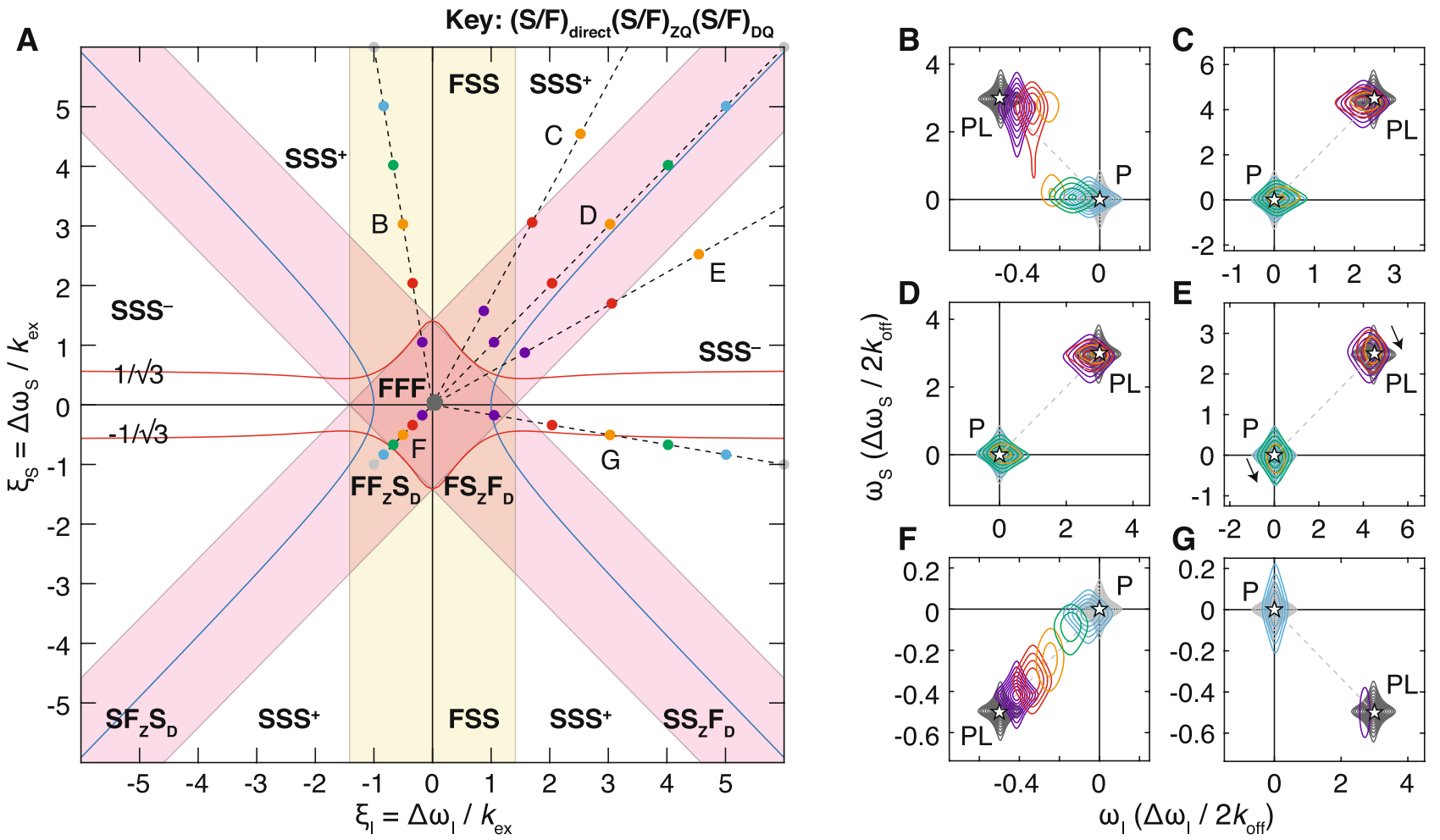

Fig. 3 Classification and examples of two-dimensional exchange regimes for multiple-quantum correlation experiments. a Two-dimensional exchange regimes are plotted as a function of the relative frequency differences $\xi_{I}=\Delta \omega_{I} / k_{\mathrm{ex}}$ and $\xi_{S}=\Delta \omega_{S} / k_{\mathrm{ex}}$. A yellow band indicates the region of fast exchange in the direct dimension (bounded by the coalescence point), while red bands indicate the region of fast exchange in the indirect dimension, corresponding to zero and double quantum frequency differences. A red line indicates the multiple-quantum coalescence point (Eq. 1), and a blue line indicates the boundary at which the initial chemical shift change (i.e. when $\left.p_{B} \ll 1\right)$ in the indirect dimension has the opposite sign to the true

initial direction of chemical shift changes over the course of a titration is hard to discern, or even occurs in the opposite direction to the bound peak position; we have labelled this regime ' $\mathrm{SS}^{-}$' (Fig. 3a). A calculation of chemical shift perturbations across all exchange regimes, in the limit $p_{B} \ll 1$, indicates that this reversal occurs when $\xi_{I}^{2}>\xi_{S}^{2}+1$. This region of parameter space is delineated with a blue line in Fig. 3b. This effect has previously been noted in the context of relaxation dispersion experiments, where the difference in chemical shifts of a resonance between HSQC and HMQC experiments can be used to infer the sign of the chemical shift difference to the unobserved excited state (Skrynnikov et al. 2002).

It is also possible to calculate the coalescence point for the indirect dimension of the HMQC experiment. In contrast to the HSQC, for which the coalescence point occurs at $\xi_{S}= \pm \sqrt{2}$, in the HMQC the coalescence point must be defined as a function of the frequency difference $\xi_{I}$ also. By calculating the time evolution of the magnetisation chemical shift difference. $\mathbf{b}-\mathbf{g}$ Simulated titration measurements for residues having relative frequency differences (at the titration midpoint): $\mathbf{b} \xi_{I}=0.5$ and $\xi_{S}=3 ; \mathbf{c} \xi_{I}=3$ and $\xi_{S}=3 ; \mathbf{d} \xi_{I}=4$ and $\xi_{S}=3$; $\mathbf{e} \xi_{I}=0.5$ and $\xi_{S}=0.5 ; \mathbf{f} \xi_{I}=3$ and $\xi_{S}=0.5$; and $\mathbf{g} \xi_{I}=3$ and $\xi_{S}=4$. Asterisks mark the positions of the free and bound resonances. Ligand concentrations were selected so that the bound fraction varied uniformly from 0 to $99.9 \%$ (grey-blue-purple-grey). Contour levels are equal for all spectra, spaced at intervals incrementing $1.4 \times$ from a base value of $2 \%$ of the maximum intensity in the apo spectrum. The relative frequency differences, varying across the titration series, are plotted in a

analytically, including the central $\pi \hat{I}_{x}$ pulse to interconvert zero and double quantum coherences, and then taking the Fourier transform of the result, we obtain the lineshape in the indirect dimension, $y\left(\xi_{S} ; \xi_{I}\right)$. The coalescence point is then defined by a vanishing second derivative, $d^{2} y / d \xi_{S}^{2}=0$, which yields:

$\xi_{I}^{2}\left(\xi_{I}^{2}-3\right) \xi_{S}^{4}-\left(3 \xi_{I}^{6}+9 \xi_{I}^{4}+19 \xi_{I}^{2}+4\right) \xi_{S}^{2}+\xi_{I}^{6}+\xi_{I}^{4}+8=0$

Solutions to this expression are plotted as red lines in Fig. 3a. Two further roots of Eq. 1 exist when $\left|\xi_{S}\right| \gtrsim 8$, but inspection of the relevant lineshapes indicates that this does not correspond to coalescence behaviour but rather to distortions in the baseline between two well-resolved resonances. As expected, when $\xi_{I}$ is zero then the coalescence point is identical to that of the HSQC, while for $\left|\xi_{I}\right|<1$ we observe that the region of parameter space below the coalescence point is well approximated by the requirement of being in fast exchange with respect to both zero and double quantum 
frequency differences (Fig. 3a). However, when $\left|\xi_{I}\right|$ becomes large then the coalescence point tends towards $\left|\xi_{S}\right|=1 / \sqrt{3}$. Moreover, direct inspection of simulated lineshapes within this regime $\left(\xi_{I} \gg 1, \xi_{S}<1 / \sqrt{3}\right)$ finds that, consistent with the limits calculated in Table 1, progressive chemical shift perturbations are not observed, and instead resonances interconvert with intensity modulations more similar to classical slow exchange behaviour (Fig. S1). Thus, for multiple quantum dimensions, we conclude that classical fast exchange behaviour is only observed within the $\mathrm{F}_{\mathrm{Z}} \mathrm{F}_{\mathrm{D}}$ regime.

To illustrate these cases further, a series of $2 \mathrm{D}$ titration spectra have been simulated (Fig. 3b-g), with relative frequency differences as indicated in Fig. 3a. In some cases, the simulated HMQC spectra are very similar to the equivalent HSQC spectra (Fig. 1). For example, HMQC spectra within the FSS regime (Fig. 3b), where $\xi_{I}$ is small, are comparable to HSQC spectra within the FS regime (Fig. 1b). Exchange within the $\mathrm{SSS}^{+}$and $\mathrm{SF}_{Z} \mathrm{~S}_{\mathrm{D}}$ regimes (Fig. 3c, d) is also similar, and comparable to that in the SS regime of HSQC spectra (Fig. 1c). However, in the $\mathrm{SSS}^{-}$regime (Fig. 3e) we observe, as predicted above, that the direction of the small chemical shift perturbations in the indirect dimension is opposite to that of the exchanging state. A comparison of exchange in the FF regime of the HSQC (Fig. 1d) with the FFF regime of the HMQC (Fig. 1f) shows that the extent of line broadening is more severe in the HMQC. Close inspection also shows that non-linear chemical shift perturbations, which are often taken to be a sign of more complex association mechanisms, are observed in early stages of the titration (within the $\mathrm{FF}_{\mathrm{Z}} \mathrm{S}_{\mathrm{D}}$ regime). Lastly, resonances within the SF regime of the HSQC (Fig. 1e) are now within the $\mathrm{SSS}^{-}$regime of the HMQC (Fig. 3g), close to the calculated coalescence point (Fig. 3a, red lines), and consequently very different spectra result, with severe line broadening but no chemical shift perturbations observed within the indirect dimension of the HMQC. In summary therefore, it is clear that more complex exchange phenomena are present within HMQC spectra, that on a practical level can result in stronger line broadening and altered chemical shift perturbations to those obtained through HSQC experiments.

\section{Longitudinal relaxation optimised pulse sequences for the measurement of heteronuclear zero and double quantum correlation spectra}

Our analysis above of exchange within HZQC and HDQC spectra has indicated the potential utility of this pair of complementary experiments for the analysis of molecular interactions. A number of pulse sequences have been described for the measurement of ZQ and DQ correlation spectra. The first observation of ZQ and DQ transitions was reported by Müller (1979), and magnitude mode experiments were later developed in which separate ZQ and DQ transitions could be isolated (Bax et al. 1983). It was also shown that ZQ and DQ transitions could be detected from the same physical experiment simply by applying different receiver phase cycling during processing, while experimental sensitivity could be enhanced using Ernst angle excitation (Bax et al. 1983). The first phase sensitive HZQC and HDQC experiments were reported by Jarvet and Allard, including gradient selected variants (Jarvet and Allard 1996), and an alternative sensitivity-enhanced HZQC experiment was later reported by the Kay group for application to methyl groups (Tugarinov et al. 2004), with only a loss of $\sqrt{2}$ in sensitivity relative to the HMQC (and less when the improved relaxation of methyl $\mathrm{ZQ}$ coherences is taken into account).

Here we present further refinements of the sensitivityenhanced HZQC experiment (Tugarinov et al. 2004). We have introduced longitudinal relaxation optimisation, applying the same principles as used for the SOFAST-HMQC experiment Schanda and Brutscher (2005). As established previously for the SOFAST-HMQC experiment, selective Ernst angle excitation of amide protons (or equally methyl protons if applied to a ${ }^{1} \mathrm{H},{ }^{13} \mathrm{C}$ correlation experiment) is expected here also to provide a large sensitivity gain through efficient longitudinal cross-relaxation with the surrounding bath of unexcited spins and by avoiding saturation of the solvent, allowing rapid repetition rates. Alternatively, where sensitivity is not limiting, this enables rapid acquisition of the experiment.

The pulse sequence and coherence transfer pathways for the HZQC and HDQC experiments are shown in Fig. $4 \mathrm{a} .{ }^{1} \mathrm{H}$ +1 and -1 coherence orders are selected during $t_{1}$ depending on the position of the $180^{\circ}$ refocusing pulse indicated by dashed lines. Selection of $\Delta p_{S}=+1$ or -1 is then required at the first $90^{\circ}$ pulse on the $S$ spin to isolate ZQ and DQ coherences. However, by storing individual steps of the phase cycle on this pulse and applying the required receiver phase during processing, both HZQC and HDQC experiments can be acquired simultaneously. Using the sensitivity enhanced approach, each of these spectra will have a factor of only $\sqrt{2}$ less sensitivity than original HMQC. Moreover, by shearing both ZQ and DQ spectra and then summing, a conventional correlation spectrum can in principle be obtained with identical sensitivity to the original HMQC (Fig. 4b).

We have validated our new experiment, which we term the SOFAST-H(Z/D)QC, by comparison with ${ }^{1} \mathrm{H},{ }^{15} \mathrm{~N}$ SOFAST-HMQC measurements of the folded protein ubiquitin, and the unfolded Y719E variant of the FLN5 filamin domain (Cabrita 2016; Waudby 2018). Analysis of the integrated amide signal intensity as a function of the inter-scan delay showed that for both samples the sensitivity of SOFAST-HZQC and SOFAST-HDQC experiments was very close to the expected factor of $\sqrt{2}$ less than the SOFAST-HMQC (Fig. 4c, d). Very similar intensities were observed in the first increments of the SOFAST-HZQC and 

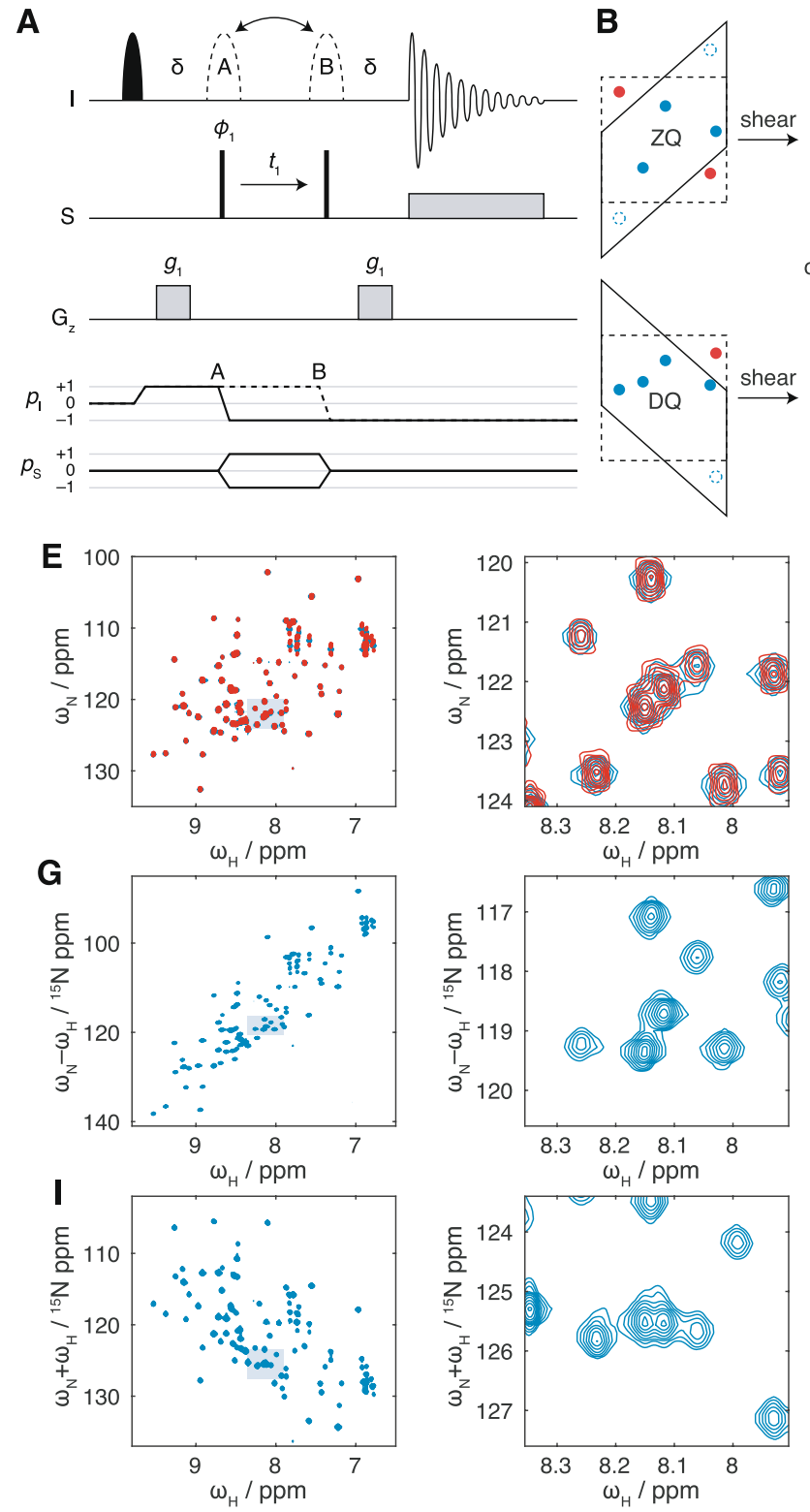

Fig. 4 The SOFAST-H(Z/D)QC experiment. a Pulse sequence and coherence transfer pathways for the SOFAST-H(Z/D)QC experiment. Two experiments are recorded for each point in $t_{1}$, in which the $180^{\circ}$ pulse indicated with dashed lines is placed at positions $\mathrm{A}$ and $\mathrm{B}$. The delay $\delta$ is $1 /(2 J)=5.5 \mathrm{~ms}$. The filled shaped pulse is a $120^{\circ}$ Pc9 pulse $(2657 \mu$ s at $8.2 \mathrm{ppm}, 700 \mathrm{MHz})$. The hollow pulses $\mathrm{A}$ and $\mathrm{B}$ are $180^{\circ}$ Reburp pulses $(1935 \mu \mathrm{s}$ at $8.2 \mathrm{ppm}, 700 \mathrm{MHz})$. Phase cycling of $\phi_{1}$ is $x,-x$ and the receiver phase $\phi_{\mathrm{rx}}$ is $x,-x$. The phase of $\phi_{1}$ is incremented by $90^{\circ}$ after each pair of experiments $\mathrm{A}$ and $\mathrm{B}$, resulting in four FIDs labelled $A_{x}, A_{y}, B_{x}$ and $B_{y}$. Cosine and sine modulated components of ZQ and DQ spectra are formed from combinations of these FIDs: $Z Q_{\cos }=A_{x}+i A_{y}+B_{x}-i B_{y}$, $Z Q_{\text {sin }}=i A_{x}-A_{y}-i B_{x}-B_{y}, \quad D Q_{\cos }=A_{x}-i A_{y}+B_{x}+i B_{y}, \quad$ and $D Q_{\text {sin }}=-i A_{x}-A_{y}+i B_{x}-B_{y}$. Note that spin dynamical phases are listed here, which when implemented may need to be adapted accord-
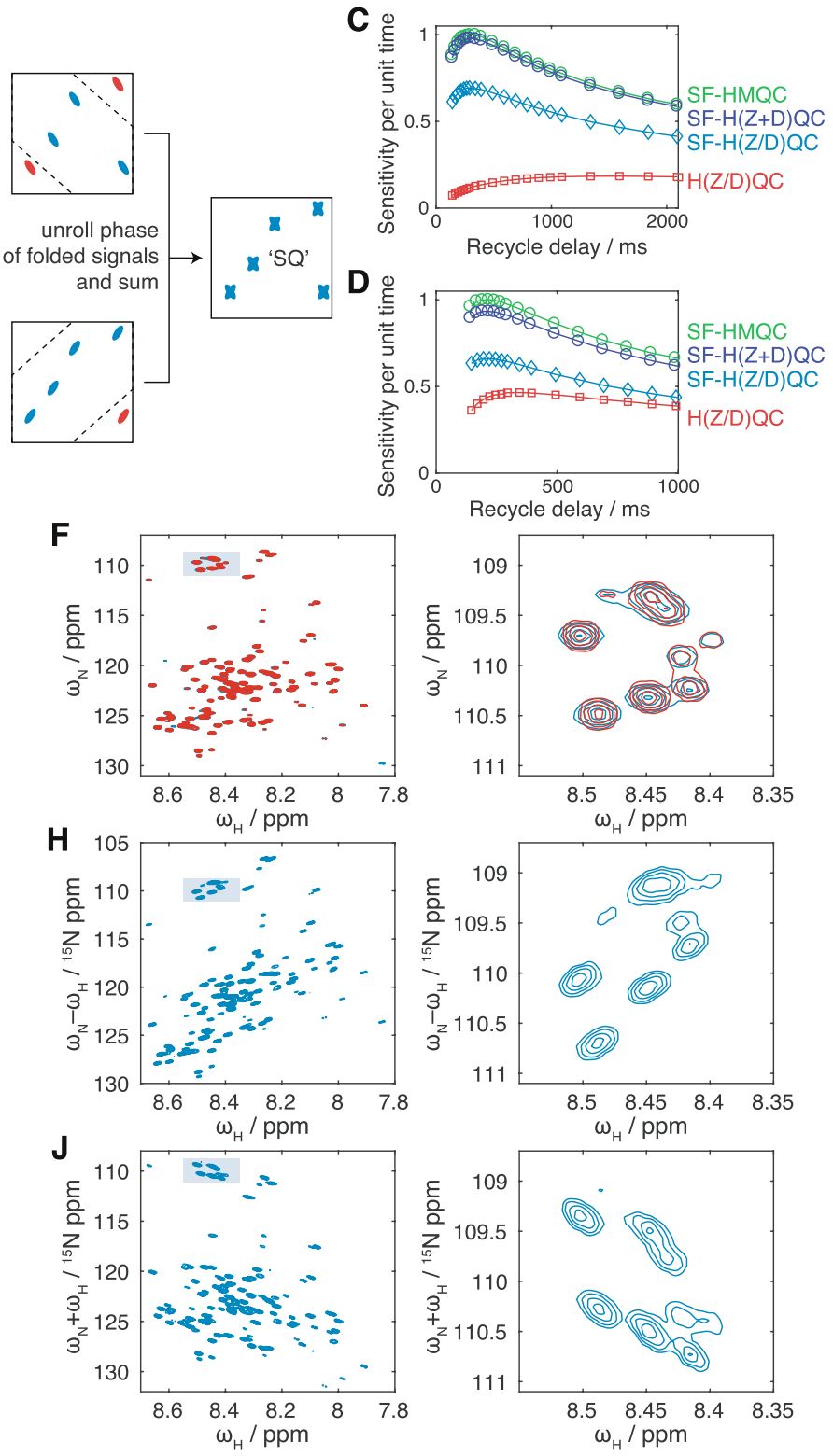

ing to the spectrometer manufacturer (Levitt 1997; Roehrl et al. 2005). b Reconstruction of single-quantum-like $H(Z+D) Q C$ spectra by shearing and recombination of component ZQ and DQ spectra. c, d Relative sensitivity, per unit experimental time, measured from the integrated intensity of the first increment of the indicated experiments as a function of the recycle delay (including the acquisition period) for $\mathbf{c}^{1} \mathrm{H},{ }^{15} \mathrm{~N}$-labelled ubiquitin $(700 \mathrm{MHz}, 298 \mathrm{~K})$ and $\mathbf{d}{ }^{1} \mathrm{H}$, ${ }^{15}$ N-labelled FLN5 Y719E (700 MHz, $283 \mathrm{~K}$ ). e, f SOFAST-HMQC (blue) and reconstructed SOFAST-H(Z+D)QC spectra (red) of e ${ }^{1} \mathrm{H},{ }^{15} \mathrm{~N}$-labelled ubiquitin $(700 \mathrm{MHz}, 298 \mathrm{~K})$ and $\mathbf{f}{ }^{1} \mathrm{H},{ }^{15} \mathrm{~N}$-labelled FLN5 Y719E $(700 \mathrm{MHz}, 283 \mathrm{~K})$. Contour levels are matched between experiments, and the magnified region is indicated by the shaded box. $\mathbf{g}-\mathbf{j}$ SOFAST-HZQC $(\mathrm{G}, \mathrm{H})$ and SOFAST-HDQC spectra $(\mathrm{I}, \mathrm{J})$ of $(\mathrm{G}, \mathrm{I}){ }^{1} \mathrm{H},{ }^{15} \mathrm{~N}$-labelled ubiquitin $(700 \mathrm{MHz}, 298 \mathrm{~K})$ and $(\mathrm{H}, \mathrm{J})$ ${ }^{1} \mathrm{H},{ }^{15} \mathrm{~N}$-labelled FLN5 Y719E (700 MHz, $283 \mathrm{~K}$ ) 
SOFAST-HDQC experiments and therefore only a single trace is shown in Fig. $4 \mathrm{c}$,d. Comparison with an equivalent $\mathrm{H}(\mathrm{Z} / \mathrm{D}) \mathrm{QC}$ experiment in which selective pulses in Fig. 4a were replaced with hard pulses shows that, as previously observed for the SOFAST-HMQC (Schanda and Brutscher 2005), a substantial increase in sensitivity (or equivalently, a reduction in acquisition time) is obtained through the optimisation of longitudinal relaxation. This effect is particularly large in the case of the unfolded protein FLN5 Y719E (Fig. 4d), indicating the importance of avoiding saturation of solvent protons. Lastly, as expected, we find that the sensitivity of the combined ZQ and DQ experiments (Fig. 4b), which we term the SOFAST-H(Z+D)QC, is only marginally less than the original SOFAST-HMQC experiment (Fig. 4c, d).

SOFAST-HMQC, HZQC and HDQC spectra of ubiquitin are presented in Fig. $4 \mathrm{e}, \mathrm{g}$, i. Note that because ${ }^{15} \mathrm{~N}$ has a negative gyromagnetic ratio, the zero quantum frequency corresponds to the sum of the chemical shifts, $\delta_{\mathrm{N}}+\delta_{\mathrm{H}}$, while the double quantum frequency corresponds to the difference in chemical shifts, $\delta_{\mathrm{N}}-\delta_{\mathrm{H}}$. A larger spectral width was used to acquire these HZQC and HDQC experiments, but in practice this could be avoided as folded signals are unlikely to overlap with other resonances. As expected, HZQC and HDQC resonances are well resolved, with symmetric lineshapes. The sheared and reconstructed SOFAST-H(Z+D) QC spectrum (Fig. 4b) is also shown in red in Fig. 4E. This overlays closely with the SOFAST-HMQC spectrum, with comparable intensity. However, due to the shearing transform of the original Lorentzian tails in the direct dimensions of the ZQ and DQ spectra, the reconstructed crosspeaks have a discernible ' $X$ ' shape, with a slightly narrower linewidth on-resonance.

Similar spectra of the unfolded protein FLN5 Y719E are presented in Fig. 4f, h, j. In this case, due to the slower transverse relaxation associated with the more flexible polypeptide chain, the ${ }^{3} J_{\mathrm{HNHA}}$ scalar coupling has a significant contribution to resonance linewidths in the direct dimension of the HMQC (Fig. 4f). Due to the amide selective refocusing pulse in the SOFAST-HMQC, this coupling is refocused during $t_{1}$. However, in the SOFAST-HZQC and HDQC experiments the use of the selective pulse gives rise to an E.COSY effect (Griesinger et al. 1987) that results in an undesirable diagonal appearance of resonances. This effect could be alleviated by deuteration of the protein at the $\mathrm{H} \alpha$ position. Indeed, this has previously been reported to greatly increase the resolution and sensitivity of more conventional HSQC and HMQC measurements of intrinsically disordered proteins (Maltsev et al. 2012).

\section{Experimental application to Hsp90 ligand binding}

Having developed a theoretical understanding of the effects of chemical exchange on HSQC, HMQC, HZQC and HDQC experiments, and developed new sensitivity optimised pulse sequences for the measurement of HZQC and HDQC
A<smiles>CCSc1nc(N)nc(I)n1</smiles>

B

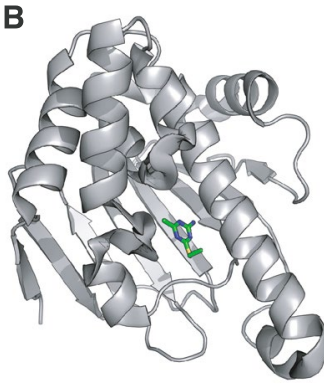

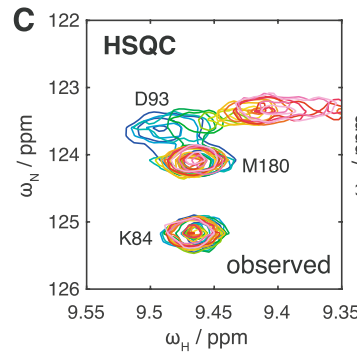

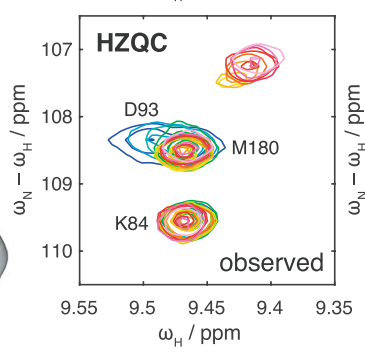

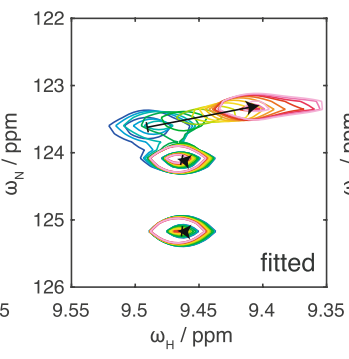
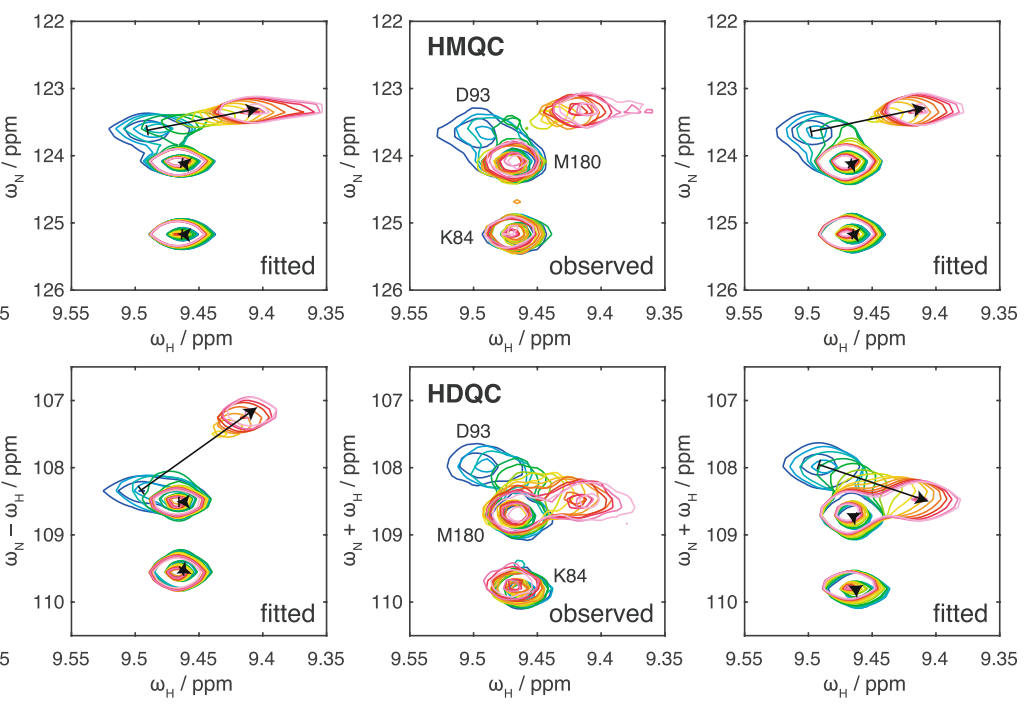

Fig. 5 Measurement and two-dimensional lineshape analysis of the interaction between Hsp90 and a small molecule ligand. a Chemical structure of compound 1 (Brough 2009; Miura 2011). b Crystal structure of compound $\mathbf{1}$ in complex with human Hsp90 alpha N-terminal domain (pdb: 3b24) (Miura 2011). c Titration of $70 \mu \mathrm{M}$ ${ }^{1} \mathrm{H},{ }^{15} \mathrm{~N}$ human Hsp90 NTD with compound 1, observed by HSQC, SOFAST-HMQC and SOFAST-H(Z/D)QC experiments as indicated, showing previously determined resonance assignments (298 K, 950 $\mathrm{MHz}$ ) (Jacobs 2006; Park et al. 2011). Ligand concentrations were 0, $7.3,14.5,29,51,80,116,159,209,273,350,441,552,674$ and 809 $\mu \mathrm{M}$ (blue to red colouring). Two-dimensional fits are shown alongside with identical contour levels. Arrows indicate the fitted positions of free and bound resonances 
spectra, we sought to demonstrate their application to the analysis of a well-characterised protein-ligand interaction. For this, we selected the interaction of the $25 \mathrm{kDa} N$-terminal domain (NTD) of human Hsp90 (Fig. 5b) with the small molecule 1 (Fig. 5a), previously identified in a fragment screen (Brough 2009) and with a $K_{\mathrm{d}}$ of $42 \mu \mathrm{M}$ previously determined using surface plasmon resonance (Miura 2011). An NMR titration was carried out using $70 \mu \mathrm{M}$ uniformly ${ }^{1} \mathrm{H},{ }^{15} \mathrm{~N}$-labelled Hsp90 NTD, and a series of fifteen HSQC, SOFAST-HMQC and SOFAST-H(Z/D)QC spectra were acquired at ligand concentrations from 0 to $800 \mu \mathrm{M}$ (Fig. 5c).

The resulting spectra illustrate experimentally the differences between the HSQC, HMQC, HZQC and HDQC experiments predicted above. This is particularly clear in the case of residue D93, which is depicted in Fig. 5c. This resonance has frequency differences $\Delta \omega_{I}=530 \mathrm{~s}^{-1}$ and $\Delta \omega_{S}=200 \mathrm{~s}^{-1}$ between the free and bound states, relative to a fitted dissociation rate (see below) of $787 \pm 19 \mathrm{~s}^{-1}$. All coherences for this residue are therefore formally in 2D 'FF' or 'FFF' fast exchange regimes (at the mid-point, $\xi_{I}=0.34, \xi_{S}=0.13$, $\xi_{Z Q}=0.46$ and $\xi_{D Q}=-0.21$ ) (Figs. 1-3). The exchange behaviour in the direct dimension is identical for all spectra, with the relatively large frequency difference giving rise to fast-intermediate exchange behaviour, i.e. line broadening, in all experiments. However, the frequency differences in the indirect dimension of HSQC and HDQC experiments are smaller, and this results in a faster exchange regime and strong cross-peaks at every point in the titration series. In contrast, the larger zero quantum frequency difference results in exchange closer to the intermediate regime, and therefore more severe line broadening in HZQC measurements. Lastly, exchange in HMQC experiments is in essence the 'worst case' of both HZQC and HDQC measurements (Fig. 3), and therefore despite the superficial similarity of the spectra to those from HSQC experiments, exchange line broadening of the D93 resonance is much more severe.

Such variations in the appearance of a single residue across multiple experiments may be a useful tool to provide independent probes of chemical exchange, that will ultimately provide improved confidence in parameters such as dissociation rates that are obtained from analysis of titration data. To explore this, we performed independent two-dimensional lineshape analyses of each set of spectra, carrying out global fits of multiple residues exhibiting a range of exchange behaviours (Figs. 5c, S2-5). Uncertainties in the fitted parameters were evaluated using both the previously described block bootstrap resampling algorithm (Waudby et al. 2016), and jackknife resampling of spins or overlapping groups of spins (see "Methods") (Table 2). Similar estimates of the dissociation constant and dissociation rate were obtained from all four sets of spectra. However, given the variation between individual fits, the bootstrap
Table 2 Hsp90 fit results

\begin{tabular}{lll}
\hline Experiment & $K_{\mathrm{d}} / \mu \mathrm{M}$ & $k_{\mathrm{off}} / \mathrm{s}^{-1}$ \\
\hline SPR Miura (2011) & 42 & N.D. \\
HSQC & $45.5 \pm 0.7_{B} \pm 2.9_{J}$ & $835 \pm 24_{B} \pm 178_{J}$ \\
HMQC & $50.2 \pm 0.5_{B} \pm 2.5_{J}$ & $787 \pm 10_{B} \pm 31_{J}$ \\
HZQC & $53.3 \pm 1.1_{B} \pm 1.8_{J}$ & $779 \pm 24_{B} \pm 24_{J}$ \\
HDQC & $55.3 \pm 0.5_{B} \pm 6.0_{J}$ & $964 \pm 14_{B} \pm 122_{J}$ \\
Combined & $51.1 \pm 1.3$ & $787 \pm 19$ \\
\hline
\end{tabular}

Uncertainties in fitted parameters calculated from two-dimensional lineshape analysis by block bootstrap resampling of residuals (Waudby et al. 2016) are indicated with a subscript $B$, while those obtained from jackknife sampling of multiple spins or spin groups (where overlapping) are indicated with a subscript $J$. The combined result shows the mean of all four NMR measurements, weighted according to the uncertainties determined by the jackknife method

N.D. not determined

method appears to have underestimated the uncertainty in these parameters. The reason for this is not clear at present. Nevertheless, using the larger error estimates determined by the jackknife approach, consistent parameter estimates were obtained across all sets of measurements. The jackknife method has been implemented in v1.6 of TITAN and on the basis of these observations, we recommend that users perform error estimates using both approaches and choose the most conservative result. The combined dissociation constant determined from these measurements, $51.1 \pm 1.3$ $\mu \mathrm{M}$, was slightly greater than the value of $42 \mu \mathrm{M}$ previously reported using SPR measurements (Miura 2011). This may reflect the effects of attachment to the surface of the biosensor, or the slightly lower ionic strength of the buffer used for SPR (50 mM Tris-based saline, $\mathrm{pH}$ 7.6, versus $50 \mathrm{mM}$ sodium phosphate, $50 \mathrm{mM} \mathrm{NaCl}$, $\mathrm{pH}$ 7.5).

\section{Longitudinal and transverse relaxation optimised HZQC and HDQC pulse sequences}

The SOFAST-H(Z/D)QC pulse sequence described above (Fig. 4a) is suitable for observations of amide resonances in intrinsically disordered proteins or folded domains with relatively low molecular weights (e.g. the $25 \mathrm{kDa} H s p 90$ NTD, Fig. 5). Perdeuteration will improve the accessible range of molecular weights further, but such systems are then most effectively observed using transverse relaxation optimised experiments, such as amide or methyl TROSY experiments. The amide SOFAST-H(Z/D)QC experiment (Fig. 4a) may be readily adapted and applied to methyl spin systems, in an analogous manner to the methyl-SOFASTHMQC experiment (Amero 2009). The HZQC has already been reported to have more favourable relaxation properties than the 'methyl-TROSY' HMQC experiment (Tugarinov et al. 2004), and using the sequence described here 
an additional HDQC spectrum may be acquired at no cost. Additional variants have been reported incorporating filters for the fast-relaxing outer lines (Gill and Palmer 2011), or in which heteronuclear polarisation may be used to enhance the sensitivity of HZQC experiments further (Ollerenshaw et al. 2005). However, two-dimensional lineshape analysis of spectra obtained using these more complex pulse sequences is not currently implemented in TITAN, and we therefore recommend the SOFAST-H(Z/D)QC sequence described above (Fig. 4a).

The case of amide spin systems is more complex. The TROSY experiment, in which the $H^{\beta} N^{ \pm}$transition is correlated with the $H^{-} N^{\beta}$ transition, has the most favourable transverse relaxation properties (Pervushin et al. 1999). However, multiple quantum coherences also have a TROSY effect due to the absence of intra-system dipolar relaxation, and due to destructive CSA-CSA interference, ZQ coherences have particularly favourable relaxation properties. However, due to presence of transverse proton magnetisation, the relaxation of both ZQ and DQ coherences by external spins is more severe than for the $H^{\beta} N^{ \pm}$TROSY transition (Pervushin et al. 1999, 1997).

A sensitivity-enhanced ZQ-TROSY experiment has previously been described (Pervushin et al. 1999). Here

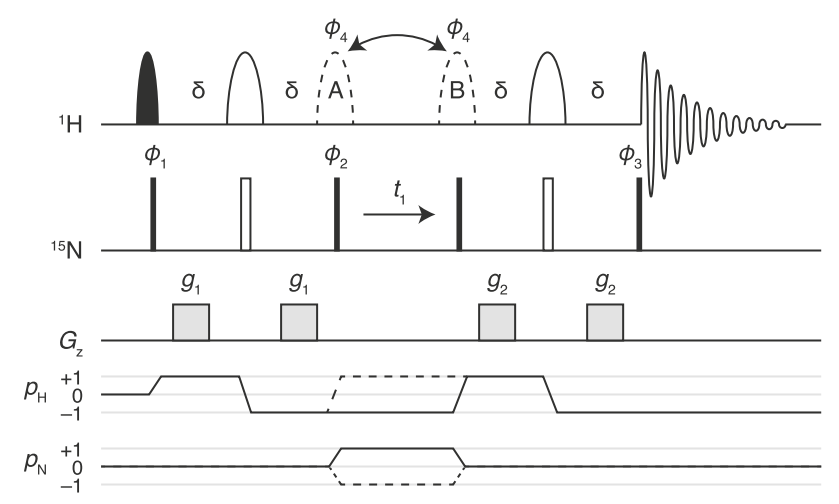

Fig. 6 Pulse sequence and coherence transfer pathways for the BEST-ZQ-TROSY experiment. The filled shaped pulse is a $90^{\circ}$ Pc9 pulse $(1958 \mu$ s at $8.2 \mathrm{ppm}, 950 \mathrm{MHz})$ while the hollow pulses are $180^{\circ}$ Reburp pulses $(1432 \mu \mathrm{s}$ at $8.2 \mathrm{ppm}, 950 \mathrm{MHz})$. The delay $\delta$ is $1 /(4 J)=2.7 \mathrm{~ms}, \phi_{3}$ is $-y, \phi_{4}$ is cycled $x_{4}, y_{4},(-x)_{4},(-y)_{4}$ and the receiver phase is cycled $x,-x,-y, y,-x, x, y,-y$. Gradients are applied as $1 \mathrm{~ms}$ trapezoidal pulses, $g_{1}=11 \%$ and $g_{2}=7 \%$. Frequency discrimination is achieved by the echo/anti-echo method. The echo (dashed CTP) is acquired with the dashed pulse at ' $\mathrm{A}$ ', $\phi_{1}-x, x,-y, y$ and $\phi_{2}-y, y,-x, x$, and the anti-echo (solid CTP) is acquired with the dashed pulse at 'B', $\phi_{1}-x, x, y,-y$ and $\phi_{2}-y, y, x,-x$. Alternatively, the BEST-DQ-TROSY experiment may be acquired by setting $\phi_{3}$ to $y$. The DQ echo is acquired with the dashed pulse at 'A', $\phi_{1}-x, x,-y, y$ and $\phi_{2}-y, y, x,-x$, and the DQ anti-echo is acquired with the dashed pulse at ' $\mathrm{B}$ ', $\phi_{1}-x, x, y,-y$ and $\phi_{2}-y, y,-x, x$. Note that spin dynamical phases are listed here, which when implemented may need to be adapted according to the spectrometer manufacturer (Levitt 1997; Roehrl et al. 2005) we present an updated BEST-ZQ-TROSY experiment that incorporates longitudinal relaxation optimisation using selective excitation of amide resonances (Fig. 6). In contrast to the SOFAST-H(Z/D)QC, it is not possible to acquire both ZQ and DQ experiments simultaneously, but a BEST-DQTROSY experiment may be acquired separately by modification of the pulse phases as described in the figure legend.

We have tested this new sequence experimentally with observations of ${ }^{2} \mathrm{H},{ }^{156} \mathrm{~N}$-labelled ubiquitin in $\mathrm{H}_{2} \mathrm{O}$ at $277 \mathrm{~K}$, $950 \mathrm{MHz}$ (Fig. 7). While the rotational correlation time is still relatively low, ca. $9 \mathrm{~ns}$, this nevertheless provides validation of the pulse sequence and points towards applications to higher molecular weight systems. Fig. $7 \mathrm{a}-\mathrm{d}$ presents a comparison of BEST-TROSY (Favier and Brutscher 2011), BEST-HSQC (Lescop et al. 2010), BEST-ZQ-TROSY and BEST-DQ-TROSY experiments, which were all acquired with identical acquisition times and parameters. Folded resonances can be observed in both ZQ and DQ experiments (magenta contours), but it is clear that this does not introduce additional resonance overlap. Cross-sections through the representative E64 resonance are shown in Fig. 7e-h, and these were fitted to Lorentzian lineshapes to determine the indicated linewidths. As expected, TROSY, ZQ-TROSY and DQ-TROSY experiments all had reduced linewidths in the direct dimension relative to the HSQC. In the indirect dimension, the ZQ resonance was marginally sharper than the HSQC resonance, and approximately 1.6 times broader than the TROSY, while the DQ resonance was substantially more broad (2.6 times that of the TROSY). However, the intensities of the TROSY and ZQ-TROSY resonances were comparable, and significantly higher than in both HSQC and DQ-TROSY spectra. Thus, we believe the BEST-ZQTROSY experiment may be a useful and reasonably sensitive experiment for applications in high molecular weight systems. Importantly, because coherence transfer in this experiment is much simpler than in the regular TROSY experiment (in which the back-transfer $H^{\beta} N^{ \pm} \rightarrow H^{-} N^{\beta}$ occurs through several stages), the ZQ-TROSY experiment (and indeed the DQ-TROSY experiment) is amenable to two-dimensional lineshape analysis.

\section{Discussion}

In this paper we have presented a systematic analysis of chemical exchange regimes within two-dimensional correlation spectra, focusing in particular on differences between SQ, MQ, ZQ and DQ-based experiments. In many ways, these results are not new. We have previously commented upon the differences in exchange behaviour between HSQC and HMQC experiments (Waudby et al. 2016), and indeed the use of ZQ and DQ TROSY experiments to reduce chemical exchange-induced resonance broadening has also been 

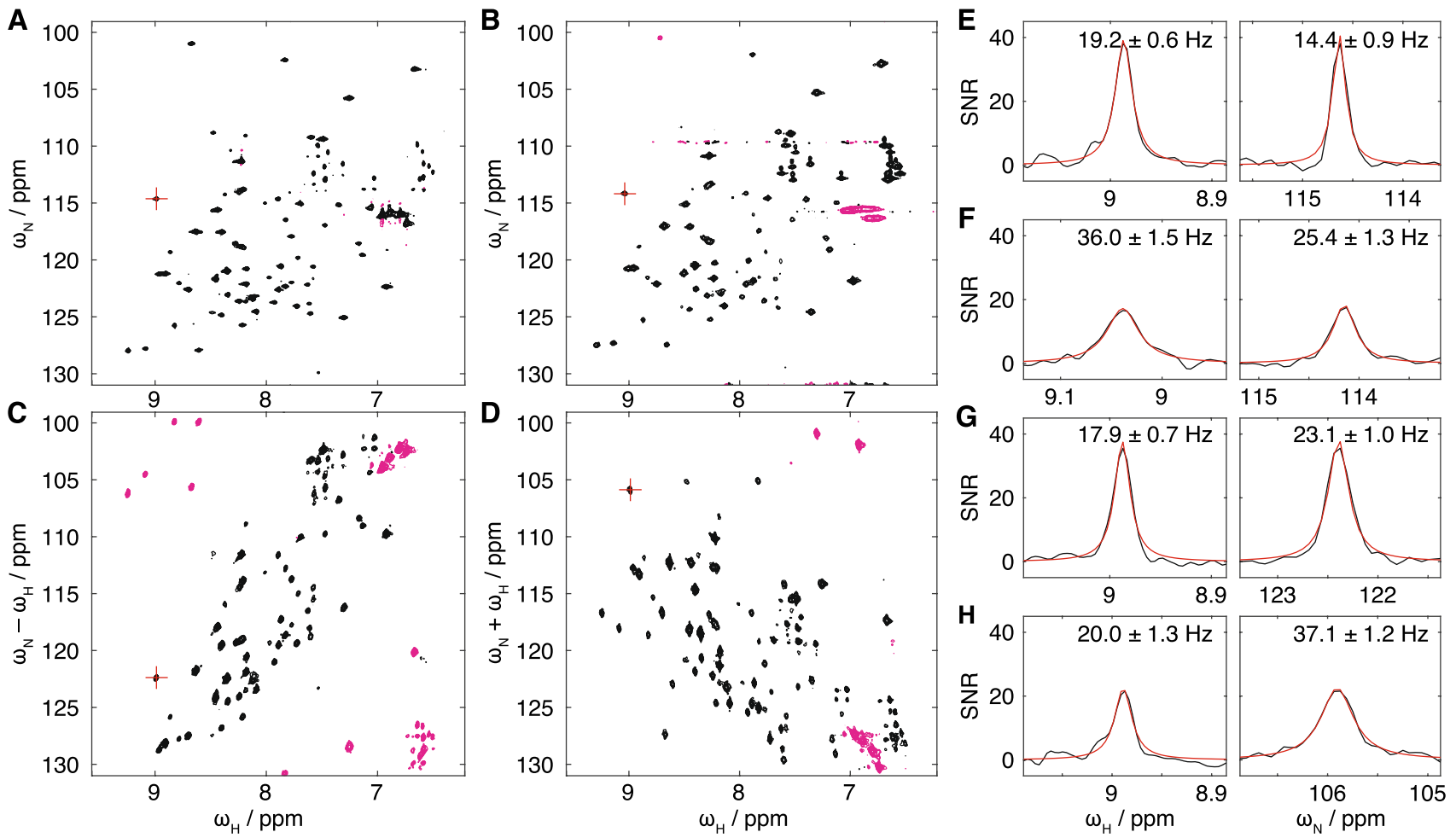

Fig. 7 a BEST-TROSY, b BEST-HSQC, c BEST-ZQ-TROSY and d BEST-DQ-TROSY spectra of ${ }^{2} \mathrm{H},{ }^{15} \mathrm{~N}$-labelled ubiquitin in $\mathrm{H}_{2} \mathrm{O}(277$ $\mathrm{K}, 950 \mathrm{MHz}$ ). Spectra were acquired with identical acquisition times and are shown with matched contour levels (magenta contours indicate negative intensities). Cross-sections through the E64 resonance, indicated with red crosshairs in a-d, are plotted for e BEST-TROSY, f BEST-HSQC, $\mathbf{g}$ BEST-ZQ-TROSY and $\mathbf{h}$ BEST-DQ-TROSY spectra. Red lines show fits to Lorentzian lineshapes with linewidths as indicated

titration measurements. As an alternative or complement, we have therefore developed the SOFAST-H(Z/D)QC experiment (Fig. 4a) for the simultaneous and sensitive acquisition of ZQ and DQ correlation spectra. Such spectra have reduced sensitivity to chemical exchange (Fig. 2) compared to the original HMQC, and this may be particularly useful in the case of titrations observed using methyl labelling. We have also developed longitudinal relaxation optimised BEST-ZQ-TROSY and BEST-DQ-TROSY experiments for application to amide spin systems (Fig. 6). While transverse relaxation of $Z Q$ coherences is not quite as optimal as in the full TROSY experiment (Fig. 7), the simpler pulse sequence means that these experiments are amenable to two-dimensional lineshape analysis, in contrast to standard TROSY experiments.

We have explored the difference between SQ, MQ, ZQ and DQ correlation experiments on experimental titration measurements by observing the interaction of the Hsp90 NTD with a small molecule ligand (Fig. 5) (Miura 2011). As expected, resonances with the HSQC, HMQC, HZQC and HDQC experiments that were acquired exhibited different chemical exchange behaviours, and the independent twodimensional lineshape analysis of the four titration series 
using TITAN ultimately provided improved confidence in the dissociation constant and dissociation rate determined (Table 2). As the additional time required to acquire a second correlation spectrum during a titration measurement is often small relative to the total time required for setup and sample handling, we suggest that this may be a useful strategy in general: either by acquiring HSQC and HMQC measurements sequentially, or acquiring HZQC and HDQC experiments simultaneously as described above using the SOFAST-H(Z/D)QC experiment.

An additional virtue of such an approach is that it provides increased sensitivity to departures from simple twostate association mechanisms. NMR lineshapes are well known to be sensitive probes of more complex binding mechanisms, such as induced fit or conformational selection (Waudby et al. 2016; Kovrigin 2012; Agafonov et al. 2014), and the independent analysis of multiple experiment series may reduce the chance of overlooking such mechanisms. Equally, where such multi-state mechanisms have been identified, the analysis of multiple experiments may provide increased confidence in the fitted parameters. Ultimately, in analogy to analyses of relaxation dispersion measurements (Orekhov et al. 2004; Dittmer and Bodenhausen 2004; Korzhnev et al. 2005), we envisage the global analysis of multiple experiment types (or indeed experiments at multiple field strengths), and efforts are underway to implement this in a future version of TITAN.

\section{Methods}

\section{Sample preparation}

Hsp90 protein was produced as described previously (Wright 2004). The N-terminal fragment of Hsp90alpha (residues 9-236) was overexpressed in the E. Coli strain BL21 (pLysS) grown in EnPresso defined media (SigmaAldrich) supplemented with $4 \mathrm{~g} / \mathrm{L}{ }^{15} \mathrm{~N}$ ammonium chloride. The construct included a deca-his tag within a 15 amino acid N-terminal extension of MGHHHHHHHHHHSSGH, and the protein was purified using a $\mathrm{Ni}^{2+}$ affinity column followed by a monoQ ion-exchange column.

Compound 1 was identified from a fragment screen against Hsp90 as described previously (Brough 2009).

\section{NMR spectroscopy}

\section{Acquisition and processing}

All NMR data were acquired using Bruker spectrometers operating with Topspin 3.5. Pulse sequences for the SOFAST-H(Z/D)QC and BEST-ZQ-TROSY experiments are provided in supporting information. NMR data were processed using nmrPipe (Delaglio 1995). SOFAST$\mathrm{H}(\mathrm{Z}+\mathrm{D}) \mathrm{QC}$ spectra were sheared and recombined in $\mathrm{nmr}-$ Pipe using the shear.M macro. Scripts and additional processing macros for the analysis of the SOFAST-H(Z/D)QC experiment are provided in supporting information.

\section{Validation of the SOFAST-H(Z/D)QC experiment}

NMR experiments were acquired at $700 \mathrm{MHz}$ to validate and assess the sensitivity of the SOFAST-H(Z/D)QC experiment using a ca. $1 \mathrm{mM}$ sample of ${ }^{1} \mathrm{H},{ }^{15} \mathrm{~N}$-labelled ubiquitin $(10 \%$ $\mathrm{D}_{2} \mathrm{O}, 298 \mathrm{~K}$ ) and a $350 \mu \mathrm{M}$ sample of ${ }^{1} \mathrm{H},{ }^{15} \mathrm{~N}$-labelled FLN5 Y719E (10\% $\left.\mathrm{D}_{2} \mathrm{O}, 283 \mathrm{~K}\right)$. SOFAST-HMQC experiments (Schanda and Brutscher 2005) were acquired using the same shaped pulses as specified for the SOFAST-H(Z/D)QC (Fig. 4a) while control measurements were recorded replacing shaped pulses with corresponding hard pulses. Sensitivity was assessed from the integrated amide intensity in the first increment of experiments recorded with a constant number of scans and a $50 \mathrm{~ms}$ acquisition time, and recycle delays varied from $50 \mathrm{~ms}$ to $2 \mathrm{~s}$. Measurements were normalised for the total acquisition time (excluding dummy scans). 2D correlation spectra were acquired with a $100 \mathrm{~ms}$ recycle delay, a $100 \mathrm{~ms}$ acquisition time (1024 complex points) in the direct dimension and a $100 \mathrm{~ms}$ acquisition time (512 complex points) in the indirect dimension.

\section{Hsp90 titration measurements}

A $70 \mu \mathrm{M}$ sample of ${ }^{1} \mathrm{H},{ }^{15} \mathrm{~N}$-labelled human Hsp90 N-terminal domain $(50 \mathrm{mM}$ sodium phosphate, $50 \mathrm{mM} \mathrm{NaCl}, \mathrm{pH}$ $7.5,10 \% \mathrm{D}_{2} \mathrm{O}$ ) was titrated with a $20 \mathrm{mM}$ stock of ligand $\mathbf{1}$ (Fig. $5 \mathrm{a}$ ) in $\mathrm{d}_{6}$-DMSO. 15 points were recorded, up to a maximum ligand concentation of $810 \mu \mathrm{M}$. NMR experiments were acquired at $298 \mathrm{~K}, 950 \mathrm{MHz}$, with a $100 \mathrm{~ms}$ acquisition time ( 1536 complex points) in the direct dimension and a 21 ms acquisition time (64 complex points) in indirect dimensions. SOFAST-HMQC (Schanda and Brutscher 2005) and SOFAST-H(Z/D)QC experiments were acquired with a recycle delay of $300 \mathrm{~ms}$, and FHSQC experiments (Mori et al. 1995) were acquired with a $1 \mathrm{~s}$ recycle delay.

\section{BEST-ZQ-TROSY and BEST-DQ-TROSY experiments}

NMR experiments were acquired at $950 \mathrm{MHz}$ to validate and assess the sensitivity of the BEST-ZQ-TROSY and BESTDQ-TROSY experiments using a ca. $1 \mathrm{mM}$ sample of ${ }^{2} \mathrm{H}$, ${ }^{15} \mathrm{~N}$-labelled ubiquitin $\left(10 \% \mathrm{D}_{2} \mathrm{O}, 277 \mathrm{~K}\right)$. BEST-TROSY spectra (Favier and Brutscher 2011) were acquired using the b_trosyf3gpph.2 library pulse sequence. Spectra were acquired with a $100 \mathrm{~ms}$ recycle delay, a $100 \mathrm{~ms}$ acquisition time (1536 complex points) in the direct dimension and an 
83 ms acquisition time (256 complex points) in the indirect dimension.

\section{Lineshape analysis}

Two-dimensional lineshape fitting, and simulations of HSQC, HMQC, HZQC and HDQC spectra, were carried out using TITAN (v1.6) (Waudby et al. 2016). HZQC and HDQC experiments have been implemented by a simple modification of the original HMQC implementation (Waudby et al. 2016) to isolate the subspace of ZQ or DQ coherences. Fitting of the Hsp90 titration data was carried out in two stages. The chemical shifts and linewidths of the unbound state were initially fitted using only the first spectrum, acquired in the absence of ligand. These parameters were then fixed, and the chemical shifts and linewidths of the bound state were fitted together with the dissociation constant and the dissociation rate. Bootstrap error estimates were calculated using 100 replicas. A second estimate of uncertainty in the dissociation constant and dissociation rate was also determined using a jackknife algorithm, in which fitting was repeated multiple times, with each spin system being omitted in turn. The reported uncertainties from this method are the standard deviation across all fits. The jackknife algorithm has been implemented in the v1.6 release of TITAN.

Acknowledgements We thank Anais Cassaignau for providing the sample of FLN5 Y719E, and Frank Delaglio for help preparing nmrPipe macros. We acknowledge the use of the UCL Biomolecular NMR Centre and the staff for their support. This work was supported by the Francis Crick Institute through provision of access to the MRC Biomedical NMR Centre. The Francis Crick Institute receives its core funding from Cancer Research UK (FC001029), the UK Medical Research Council (FC001029), and the Wellcome Trust (FC001029). This work was supported by a Wellcome Trust Investigator Award (to J.C., 206409/Z/17/Z).

\section{Compliance with ethical standards}

Conflict of interest The authors declare no competing financial interests.

Open Access This article is licensed under a Creative Commons Attribution 4.0 International License, which permits use, sharing, adaptation, distribution and reproduction in any medium or format, as long as you give appropriate credit to the original author(s) and the source, provide a link to the Creative Commons licence, and indicate if changes were made. The images or other third party material in this article are included in the article's Creative Commons licence, unless indicated otherwise in a credit line to the material. If material is not included in the article's Creative Commons licence and your intended use is not permitted by statutory regulation or exceeds the permitted use, you will need to obtain permission directly from the copyright holder. To view a copy of this licence, visit http://creativecommons.org/licenses/by/4.0/.

\section{References}

Agafonov RV, Wilson C, Otten R, Buosi V, Kern D (2014) Energetic dissection of Gleevec's selectivity toward human tyrosine kinases. Nat Struct Mol Biol 21:848-853

Amero C et al (2009) Fast two-dimensional NMR spectroscopy of high molecular weight protein assemblies. J Am Chem Soc 131:3448-3449

Bain AD (2003) Chemical exchange in NMR. Prog Nucl Magn Reson Spectrosc 43:63-103

Bax A, Griffey RH, Hawkins BL (1983) Correlation of proton and nitrogen-15 chemical shifts by multiple quantum NMR. J Magn Reson 1969(55):301-315

Binsch G (1969) Unified theory of exchange effects on nuclear magnetic resonance line shapes. J Am Chem Soc 91:1304-1309

Bodenhausen G, Ruben DJ (1980) Natural abundance nitrogen-15 NMR by enhanced heteronuclear spectroscopy. Chem Phys Lett 69:185-189

Brough PA et al (2009) Combining hit identification strategies: fragment-based and in silico approaches to orally active 2-aminothieno[2,3-d]pyrimidine inhibitors of the Hsp90 molecular chaperone. J Med Chem 52:4794-4809

Cabrita LD et al (2016) A structural ensemble of a ribosome-nascent chain complex during cotranslational protein folding. Nat Struct Mol Biol 23:278-285

Cousin SF et al (2016) Recovering invisible signals by two-field NMR spectroscopy. Angew Chem Int Ed Engl 55:9886-9889

Danhart EM et al (2017) Conformational and chemical selection by a trans-acting editing domain. Proc Natl Acad Sci USA 114:E6774-E6783

Delaglio F et al (1995) NMRPipe: a multidimensional spectral processing system based on UNIX pipes. J Biomol NMR 6:277-293

Dittmer J, Bodenhausen G (2004) Evidence for slow motion in proteins by multiple refocusing of heteronuclear nitrogen/proton multiple quantum coherences in NMR. J Am Chem Soc 126:1314-1315

Favier A, Brutscher B (2011) Recovering lost magnetization: polarization enhancement in biomolecular NMR. J Biomol NMR 49:9-15

Fawzi NL, Ying J, Ghirlando R, Torchia DA, Clore GM (2011) Atomic-resolution dynamics on the surface of amyloid- $\beta$ protofibrils probed by solution NMR. Nature 480:268-272

Gill ML, Palmer AG (2011) Multiplet-filtered and gradient-selected zero-quantum TROSY experiments for $13 \mathrm{C} 1 \mathrm{H} 3$ methyl groups in proteins. J Biomol NMR 51:245-251

Gopalan AB, Hansen DF, Vallurupalli P (2018) CPMG experiments for protein minor conformer structure determination. Methods Mol Biol 1688:223-242

Greenwood AI et al (2011) Complete determination of the Pin 1 catalytic domain thermodynamic cycle by NMR lineshape analysis. J Biomol NMR 51:21-34

Griesinger C, Sorensen OW, Ernst RR (1987) Practical aspects of the E. COSY technique. Measurement of scalar spin-spin coupling constants in peptides. J Magn Reson 75:474-492

Günther UL, Schaffhausen B (2002) NMRKIN: simulating line shapes from two-dimensional spectra of proteins upon ligand binding. J Biomol NMR 22:201-209

Jacobs DM et al (2006) NMR backbone assignment of the N-terminal domain of human HSP90. J Biomol NMR 36(Suppl 1):52-52

Jarvet J, Allard P (1996) Phase-sensitive two-dimensional heteronuclear zero- and double-quantum-coherence spectroscopy. J Magn Reson Ser B 112:240-244

Kloiber K, Konrat R (2000) Differential multiple-quantum relaxation arising from cross-correlated time-modulation of isotropic chemical shifts. J Biomol NMR 18:33-42

Korzhnev DM, Neudecker P, Mittermaier A, Orekhov VY, Kay LE (2005) Multiple-site exchange in proteins studied with a suite 
of six NMR relaxation dispersion experiments: an application to the folding of a Fyn SH3 domain mutant. J Am Chem Soc 127:15602-15611

Kovermann M, Rogne P, Wolf-Watz M (2016) Protein dynamics and function from solution state NMR spectroscopy. Quart Rev Biophys 49:11348

Kovrigin EL (2012) NMR line shapes and multi-state binding equilibria. J Biomol NMR 53:257-270

Lescop E, Kern T, Brutscher B (2010) Guidelines for the use of bandselective radiofrequency pulses in hetero-nuclear NMR: example of longitudinal-relaxation-enhanced BEST-type $1 \mathrm{H}-15 \mathrm{~N}$ correlation experiments. J Magn Reson 203:190-198

Levitt MH (1997) The signs of frequencies and phases in NMR. J Magn Reson 126:164-182

Maltsev AS, Grishaev A, Bax A (2012) Monomeric $\alpha$-synuclein binds Congo Red micelles in a disordered manner. Biochemistry 51:631-642

Massi F, Peng JW (2018) Characterizing protein dynamics with NMR R $1 \rho$ relaxation experiments. Methods Mol Biol 1688:205-221

McConnell HM (1958) Reaction rates by nuclear magnetic resonance. J Chem Phys 28:430-431

McShan AC et al (2018) Peptide exchange on MHC-I by TAPBPR is driven by a negative allostery release cycle. Nat Chem Biol $14: 811-820$

Miura $\mathrm{T}$ et al (2011) Lead generation of heat shock protein 90 inhibitors by a combination of fragment-based approach, virtual screening, and structure-based drug design. Bioorg Med Chem Lett 21:5778-5783

Mori S, Abeygunawardana C, Johnson MO, van Zijl PC (1995) Improved sensitivity of HSQC spectra of exchanging protons at short interscan delays using a new fast HSQC (FHSQC) detection scheme that avoids water saturation. J Magn Reson Ser B 108:94-98

Müller L (1979) Sensitivity enhanced detection of weak nuclei using heteronuclear multiple quantum coherence. J Am Chem Soc 101:4481-4484

Ollerenshaw JE, Tugarinov V, Skrynnikov NR, Kay LE (2005) Comparison of $13 \mathrm{CH} 3,13 \mathrm{CH} 2 \mathrm{D}$, and $13 \mathrm{CHD} 2$ methyl labeling strategies in proteins. J Biomol NMR 33:25-41

Orekhov VY, Korzhnev DM, Kay LE (2004) Double- and zero-quantum NMR relaxation dispersion experiments sampling millisecond time scale dynamics in proteins. J Am Chem Soc 126:1886-1891

Palmer AG (2014) Chemical exchange in biomacromolecules: past, present, and future. J Magn Reson 241:3-17

Palmer AG, Kroenke CD, Loria JP (2001) Nuclear magnetic resonance methods for quantifying microsecond-to-millisecond motions in biological macromolecules. Methods Enzymol 339:204-238

Park SJ, Kostic M, Dyson HJ (2011) Dynamic interaction of Hsp90 with Its client protein p53. J Mol Biol 411:158-173

Peng JW, Lepre CA, Fejzo J, Abdul-Manan N, Moore JM (2001) Nuclear magnetic resonance-based approaches for lead generation in drug discovery. Methods Enzymol 338:202-230

Pervushin K (2001) The use of TROSY for detection and suppression of conformational exchange NMR line broadening in biological macromolecules. J Biomol NMR 20:275-285

Pervushin K, Riek R, Wider G, Wüthrich K (1997) Attenuated T2 relaxation by mutual cancellation of dipole-dipole coupling and chemical shift anisotropy indicates an avenue to NMR structures of very large biological macromolecules in solution. Proc Natl Acad Sci USA 94:12366-12371
Pervushin KV, Wider G, Riek R, Wüthrich K (1999) The 3D NOESY[(1)H, (15)N, (1)H]-ZQ-TROSY NMR experiment with diagonal peak suppression. Proc Natl Acad Sci USA 96:9607-9612

Pons M, Millet O (2001) Dynamic NMR studies of supramolecular complexes. Prog Nucl Magn Reson Spectrosc 38:267-324

Roehrl MHA, Heffron GJ, Wagner G (2005) Correspondence between spin-dynamic phases and pulse program phases of NMR spectrometers. J Magn Reson 174:325-330

Schanda P, Brutscher B (2005) Very fast two-dimensional NMR spectroscopy for real-time investigation of dynamic events in proteins on the time scale of seconds. J Am Chem Soc 127:8014-8015

Schneider R, Blackledge M, Jensen MR (2018) Elucidating binding mechanisms and dynamics of intrinsically disordered protein complexes using NMR spectroscopy. Curr Opin Struct Biol 54:10-18

Skrynnikov NR, Dahlquist FW, Kay LE (2002) Reconstructing NMR spectra of "invisible" excited protein states using HSQC and HMQC experiments. J Am Chem Soc 124:12352-12360

Srb P et al (2019) Capturing a dynamically interacting inhibitor by paramagnetic NMR spectroscopy. Phys Chem Chem Phys 21:5661-5673

Toyama $Y$ et al (2017) Dynamic regulation of GDP binding to G proteins revealed by magnetic field-dependent NMR relaxation analyses. Nat Commun 8:14523-15

Toyama Y, Osawa M, Yokogawa M, Shimada I (2016) NMR method for characterizing microsecond-to-millisecond chemical exchanges utilizing differential multiple-quantum relaxation in high molecular weight proteins. J Am Chem Soc 138:2302-2311

Tugarinov V, Sprangers R, Kay LE (2004) Line narrowing in methylTROSY using zero-quantum $1 \mathrm{H}-13 \mathrm{C}$ NMR spectroscopy. J Am Chem Soc 126:4921-4925

Vallurupalli P, Bouvignies G, Kay LE (2012) Studying, "invisible" excited protein states in slow exchange with a major state conformation. J Am Chem Soc 134:8148-8161

Wang C, Palmer AG (2002) Differential multiple quantum relaxation caused by chemical exchange outside the fast exchange limit. J Biomol NMR 24:263-268

Waudby CA et al (2018) Systematic mapping of free energy landscapes of a growing filamin domain during biosynthesis. Proc Natl Acad Sci USA 115:9744-9749

Waudby CA, Ramos A, Cabrita LD, Christodoulou J (2016) Twodimensional NMR lineshape analysis. Sci Rep 6:24826

Waudby CA, Frenkiel T, Christodoulou J (2019) Cross-peaks in simple 2D NMR experiments from chemical exchange of transverse magnetization. Angew Chem Int Ed Engl 58:8784-8788

Wright L et al (2004) Structure-activity relationships in purine-based inhibitor binding to HSP90 isoforms. Chem Biol 11:775-785

Xue Y et al (2015) Characterizing RNA excited states using NMR relaxation dispersion. Methods Enzymol 558:39-73

Yuwen T, Kay LE, Bouvignies G (2018) Dramatic decrease in CEST measurement times using multi-site excitation. ChemPhysChem 19:1707-1710

Publisher's Note Springer Nature remains neutral with regard to jurisdictional claims in published maps and institutional affiliations. 